\title{
Teorías implícitas sobre comprensión textual y la competencia lectora de estudiantes de primer año de la Universidad de Magallanes*
}

\author{
The implicit theories about text comprehension and the reading competency \\ of the first year students at Universidad de Magallanes \\ As teorias implícitas sobre a comprensão de textos e a competencia leitora \\ de estudantes de primeiro ano da Universidade de Magallanes
}

\author{
Margarita Makuc ${ }^{1}$
}

${ }^{1}$ Universidad de Magallanes, Departamento de Educación, casilla 113-D, Punta Arenas

\begin{abstract}
RESUMEN
En este artículo se aborda el estudio de las teorías implícitas respecto de la comprensión textual de los estudiantes universitarios y su relación con los niveles de competencia lectora. Las teorías implícitas adquieren relevancia, por cuanto ellas orientarían las acciones de los sujetos interactuando con la competencia lectora, restringiendo o activando diversas estrategias del lector. Con la finalidad de identificar las teorías implícitas de los estudiantes se aplicó el cuestionario de teorías implícitas sobre comprensión textual (Makuc, 2009). La competencia lectora de los estudiantes se abordó a partir de una batería de pruebas de comprensión textual (Parodi, 2005). Este estudio nos permitió establecer el predominio de dos teorías implícitas sobre la comprensión: Interactiva y Literaria, en relación con la competencia lectora. Los estudiantes que alcanzaron superiores niveles de logro fueron aquellos que adherían a la teoría implícita interactiva.
\end{abstract}

Palabras clave: comprensión de textos, teorías implícitas, competencia lectora.

\begin{abstract}
This article is about the study of the implicit theories regarding the text comprehension of university students and its relationship with the level of reading competence. The implicit theories become relevant because they would guide the actions of the individuals interacting with the reading competence, restricting or activating different reading strategies. To identify the implicit theories of the students a questionnaire of implicit theories on text comprehension was applied (Makuc, 2009). The reading competence of the students was dealt with a battery of tests on text comprehension (Parodi, 2005). This study enabled us to establish the prevalence of two implicit theories about the comprehension: the interactive and the literary; in relation to the reading competence. The students who achieved higher levels were those who adhered to the interactive implicit theory.
\end{abstract}

Key words: text comprehension, implicit theories, reading competence.

\section{RESUMO}

Neste artigo aborda-se o estudo das teorias implícitas com respeito ao entendimento textual dos estudantes universitários e sua relação com os níveis de competência leitora. As teorias implícitas adquerem relevância porquanto elas orientariam as ações dos sujeitos interatuando com a competência leitora, restringindo ou ativando diversas estratégias do leitor. Com a finalidade de identificar as teorias implícitas dos estudantes aplicou-se o questionário de teorias implícitas sobre entendimento textual (Makuc, 2009). A competência leitora dos estudantes abordou-se a partir de uma batería de provas de comprensao textual (Parodi, 2005). Este estudo permitiu-nos estabelecer o predomínio de duas teorias implícitas sobre a comprensão: Interativa e Literária; em relação com a competência leitora. Os estudantes que atingiram níveis superiores de rendimento foram aqueles que aderiam à teoria implícita interativa.

Palavras-chave: comprensão de textos, teorias implícitas, competência leitora.

* Estudio iniciado en el marco de un proyecto de investigación interno UMAG/2008 y continuado a través del Programa de Investigación UMAG/2010-2011. Dirección de investigación Universidad de Magallanes. Resultados preliminares presentados en el V Congreso Internacional de la Cátedra UNESCO, Caracas, junio 2009. 


\section{INTRODUCCION}

En la perspectiva de profundizar en los actuales conocimientos sobre la comprensión de textos escritos y dada la necesidad de conocer los múltiples factores asociados al desarrollo de la capacidad de comprensión, en esta investigación se incorporan recientes enfoques conceptuales orientados a estudiar las teorías implícitas que sostienen los sujetos sobre diversos dominios de la vida cotidiana (Murphy y Medin 1985; Dann, 1990; Carey y Spelke, 1993; Dienes \& Perner, 1999; Pozo, 2001; Rodrigo \& Correa, 2001). Estas teorías han sido definidas como constructos epistémicos de representación de la realidad, y en consecuencia orientan las decisiones/acciones de los sujetos en múltiples ámbitos de la vida cotidiana (Karmiloff Smith, 1994, 1997; Reber \& Reber, 1999). Por ello, las teorías implícitas adquieren relevancia en este estudio, pues, desde nuestra perspectiva, forman parte de los diversos factores asociados a la comprensión textual y a las estrategias lectoras que asumen los sujetos con distintos niveles de competencia lectora/textual.

A modo de hipótesis de trabajo, interesa destacar el hecho de que los altos niveles de comprensión lectora y las estrategias consideradas eficaces se logran a partir de una permanente (re)elaboración de la experiencia por parte de los sujetos, dando con ello origen a teorías implícitas sobre la comprensión lectora, las que a su vez reflejan la influencia de los modelos culturales predominantes en un determinado contexto social. De este modo, las teorías implícitas constituyen síntesis de conocimiento que los sujetos han elaborado en determinados dominios del conocimiento/acción en el mundo (Furnham, 1988, 1990; Groeben, 1990; Dann, 1990; Mitchell, 1995).

\section{ANTECEDENTES DEL ESTUDIO Y PROBLEMA DE INVESTIGACION}

En el marco de la problemática de la comprensión textual podemos identificar una serie de antecedentes relevantes para nuestro estudio; uno de ellos dice relación con los resultados obtenidos en el Programa Internacional para la Evaluación de Estudiantes de la OCDE (PISA, 2000; 2003 y 2006) ${ }^{1}$. En todas estas mediciones Chile ha logrado bajos resultados en las tres áreas de medición: Matemáticas, Ciencias y Lectura. En este contexto, son especialmente preocupantes los déficit de habilidades y conocimientos en lectura y comprensión textual. Pese a que Chile ha mostrado un incremento significativo en lectura entre los años 2000 y 2006 (+33 puntos), aún se ubica bajo el puntaje promedio que logran el conjunto de países del OCDE (2006). En este contexto nuestro país alcanza 442 puntos, frente al promedio obtenido por los países del OECD de 492; esta distancia se incrementa significativamente respecto de los países que alcanzan los puntajes más altos como se muestra en la siguiente imagen:

1 PISA: Programme International Student Assessment. 


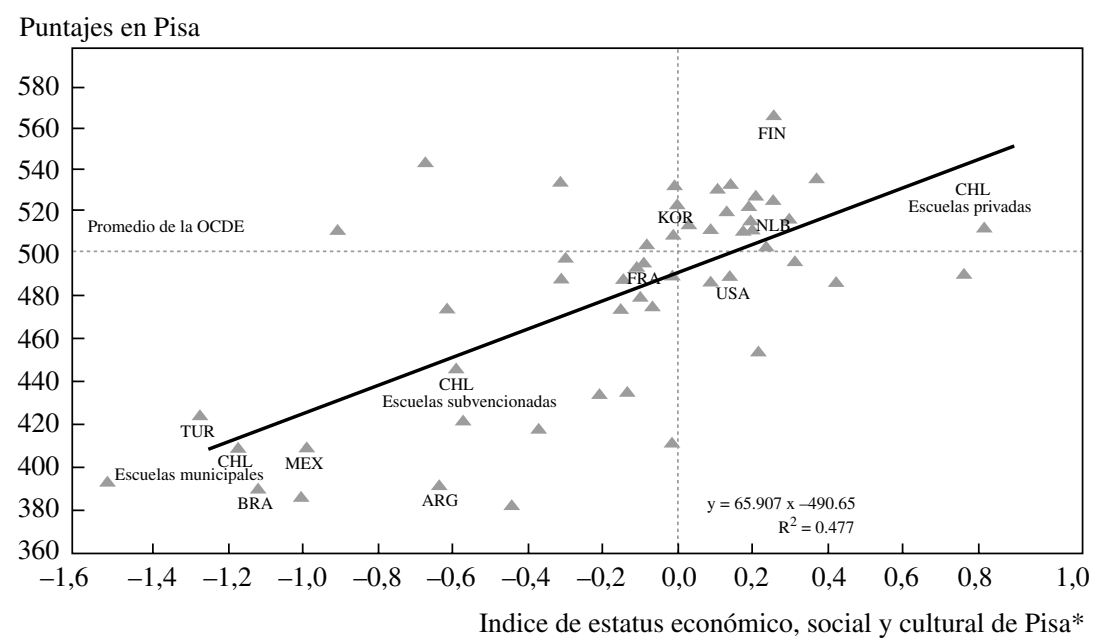

* El índice de estatus económico, social y cultural de PISA (ESCS) sintetiza varios aspectos del trasfondo socioeconómico, entre los que se encuentran el nivel de escolaridad de los padres, su categoría profesional y el acceso de los alumnos a los recursos educativos. Se normaliza a 0 para la media de la OCDE. Un valor de índice mayor es sinónimo de un contexto socioeconómico más elevado.

Fuente: OCDE Resultados de PISA 2006.

Estos resultados se han visto corroborados en el contexto nacional y los resultados de investigación indican que los sujetos adultos presentan un bajo nivel de habilidades relacionadas con el lenguaje escrito, afectando especialmente a los jóvenes que se encuentran en los diversos niveles de enseñanza del sistema educativo (Parodi, 2007; Peronard, 2007; Eyzaguirre \& Fontaine, 2008). Esta "crisis de lectura" resulta altamente preocupante para las escuelas si consideramos que la lectura no sólo es un medio básico para adquirir información, sino que principalmente constituye una competencia transversal en el aprendizaje de los estudiantes (Jamet, 2005; Regueiro, 2005).

En este contexto de análisis se considera relevante profundizar el conocimiento actual sobre la comprensión de textos escritos y de este modo aproximarse a los múltiples factores asociados al desarrollo de la capacidad de comprensión de los estudiantes universitarios de primer año en la Región de Magallanes.

\section{REFERENTES TEORICOS DE LA INVESTIGACION NOCION DE COMPRENSION TEXTUAL}

Es importante destacar que los principales estudios sobre comprensión textual así como de los factores que inciden en la experticia lectora se han desarrollado en el marco de la investigación Psicolingüística, centrando su atención en los procesos cognitivos y lingüísticos específicos que intervienen en la comprensión de un texto. De esta manera, los estudios se han orientado a la identificación de elementos como la coherencia semántica y sintáctica; la macro y microestructura de un texto (van Dijk, 1995, 1999, 2001) la generación de inferencias y la caracterización de ellas (Graesser, Singer \& Trabasso, 
1994; Paris, 1994; Colomer y Camps, 1996; Parodi, 2002; 2005ª; Rinck, Gómez, Díaz \& de Vega, 2003; Kintsch, 1994, 2002). Al mismo tiempo, una serie de estudios han enfatizado la importancia del desarrollo de estrategias lectoras (cognitivas y metacognitivas) en los sujetos y su incidencia en la competencia lectora (Mateos, 2001; Maturano et al. 2006; Peronard, 2005, 2007; Rivera, 2000; Silvestri, 2006). Desde esta perspectiva la comprensión de textos se conceptualiza como un proceso cognitivo constructivo e intencionado en el cual el lector elabora una interpretación y una representación mental de los significados textuales, basándose en la información del texto y en sus conocimientos previos, de acuerdo con un objetivo de lectura (Kintsch \& van Dijk, 1978; Smith, 1983; Laberge \& Samuels, 1994; Kintsch, 1994; van Dijk, 1995; Rumelhart, 1997; McKoon \& Ratcliff, 1998; Long \& Chong, 2001; De Vega, 2002).

En Chile, los principales estudios sobre comprensión textual se han desarrollado en el marco de la investigación psicolingüística, disciplina que ha avanzado en el conocimiento de este fenómeno centrando su atención en los procesos cognitivos específicos que intervienen en la comprensión de un texto. Para diversos autores (Parodi, 2002; 2003, 2005 a 2009; Peronard, 2005, 2007) la comprensión lingüística tiene una doble dimensionalidad: es un acto mental y un acto lingüístico simultáneamente. Estos autores, desde hace largo tiempo, han concebido el proceso de comprensión como una actividad guiada y controlada por el propio lector a la luz de sus visiones de mundo e influida por los contextos situacionales en que se desarrolla la actividad comprensiva.

Para sintetizar las bases conceptuales de esta línea de investigación se presenta una reciente definición que expone los principales supuestos de este enfoque:

\begin{abstract}
"Definimos la comprensión de un texto como un proceso cognitivo constructivo e intencionado en el que el lector elabora una interpretación y una representación mental de los significados textuales, basándose tanto en la información del texto escrito como en sus conocimientos previos y de acuerdo con un objetivo de lectura acorde a sus propósitos y a las demandas del medio social... Este proceso se plasma en una representación mental construida progresivamente sobre la base de inferencias automáticas y fundamentales para establecer la coherencia de base y se continúa reelaborando como proceso de aprendizaje a partir del texto y de los conocimientos previos a través del cual se generan múltiples procesos inferenciales y se construyen conocimientos diversos de tipo relacional" (Parodi, 2009: 39).
\end{abstract}

Sintéticamente, esta perspectiva teórica considera la comprensión textual como un proceso cognitivo complejo, que se "materializa" en una representación del discurso escrito y que es resultado de mecanismos inferenciales, estrategias cognitivas y lingüísticas que el lector activa de un modo estratégico y en interacción tanto con el texto, el medio social y sus conocimientos previos. Adicionalmente, se puede observar que en esta concepción de la actividad comprensiva intervienen una diversidad de variables asociadas al lector (conocimientos previos, concepciones de mundo, estrategias lectoras, etc.), al contexto (entornos, contextos situacionales, niveles de escolaridad, entre otras) y variables directamente asociadas al texto, en tanto objeto de actividad comprensiva (tipos de textos, rasgos lingüísticos, estructura textual, etc.)

No obstante la relevancia de estos estudios, y su incidencia en determinar aquellos aspectos psicolingüísticos asociados a la experticia lectora, nos parece central complementarlos a partir de la consideración de aspectos vinculados con las creencias que las 
personas sostienen acerca de este dominio y el modo en que estas creencias inciden en sus decisiones al momento de enfrentar un texto con la finalidad de comprenderlo. Ello implica considerar que tanto las estrategias cognitivas como metacognitivas estarían sustentadas en una teoría implícita de la comprensión textual que guiaría y orientaría las acciones y estrategias de los lectores.

\section{TEORIAS IMPLICITAS Y COMPRENSION TEXTUAL: UN ENFOQUE DESDE LAS CONCEPCIONES DEL LECTOR}

La noción de teoría implícita nos permite abordar la comprensión con un enfoque que integra en el análisis de la comprensión textual la perspectiva de los propios lectores (su experiencia formativa, sus concepciones), pues ellos, desde contextos particulares, se enfrentan a problemas y resuelven demandas específicas de comprensión textual y lo hacen activando no sólo procesos cognitivos y metacognitivos, sino además orientados por teorías implícitas transmitidas durante su proceso de formación. En consecuencia, las teorías implícitas cumplirían la función de mediar la interacción que los lectores establecen con los textos expandiendo o restringiendo los niveles de competencia lectora que éstos alcanzan en situaciones concretas.

Desde nuestra propia perspectiva de análisis, y centrándonos en la dimensión del lector, consideramos que las concepciones de mundo pueden ser conceptualizadas como teorías implícitas, es decir, constructos del pensamiento social que modulan o median tanto el conocimiento como la acción de los sujetos, jugando un rol clave en los procesos de comprensión textual.

Las teorías implícitas, definidas como constructos epistémicos de representación de la realidad que orientan y están en la base de las decisiones y acciones de los sujetos (Rodrigo, 1985; 1993; Furnham, 1988, 1990; Groeben, 1990; Dann, 1990; Mitchell, 1995; Castro, 2006), adquieren sentido para nuestro estudio, por cuanto nos interesa destacar el hecho de que ellas se elaboran a partir de la experiencia y reflejan la influencia de diversos modelos culturales, es decir, constituyen síntesis de conocimiento que los sujetos han elaborado social e individualmente respecto a un dominio específico del mundo. Por otra parte, se afirma que las teorías que los sujetos poseen acerca de un determinado dominio orientan su acción y constituyen síntesis de conocimiento que se activan a partir de demandas del contexto; en este sentido pueden considerarse estructuras mentales complejas que constituyen dominios representados mentalmente tanto de fenómenos como de sus principios explicativos (Rodrigo, 1993, 1994b, 1997; Carey y Spelke, 1993; Rodrigo, 2001).

En términos generales, la importancia de las teorías implícitas radicaría en el hecho de que son un tipo de entidad cognitiva, inconsciente, latente e implícita, que ejercen una enorme influencia en las conductas, las decisiones y en acciones o respuestas de los sujetos a determinados problemas de conocimiento (Karmiloff Smith, 1994, 1997; Kihlstrom, 1999).

En consecuencia, nos parece central complementar los estudios sobre comprensión textual integrando aspectos vinculados con las creencias o teorías implícitas que las personas sostienen acerca de este dominio y el modo en que estas creencias inciden en sus decisiones al momento de enfrentar un texto con la finalidad de comprenderlo. La relevancia que presentan las teorías implícitas al guiar a los lectores en sus decisiones 
se ha visto corroborada en diversos estudios (Schraw \& Bruning, 1996; Schraw, 2000; Peronard, Crespo, Velásquez, 2000, 2002; Makuc, 2004, 2008, 2009; Hernández, 2008). Estos estudios han permitido determinar que los lectores pueden agruparse en torno a determinadas teorías sobre la comprensión textual. Desde esta perspectiva, es posible suponer que tanto las estrategias cognitivas como metacognitivas estarían sustentadas en una teoría implícita de la comprensión textual que guiaría y orientaría las acciones de los lectores. De este modo, la noción de teoría implícita (Pozo, 2001; Rodrigo \& Correa, 2001; Rodríguez, 2001; Jiménez, 2002; Makuc, Judikis, Estrada \& Molina, 2009) nos permite abordar la comprensión con un enfoque integrador que nos posiblita centrar el análisis desde la perspectiva de los propios lectores, quienes desde contextos específicos se enfrentan a problemas y resuelven demandas específicas de comprensión textual y lo hacen activando no sólo procesos cognitivos y metacognitivos, sino además orientados por teorías implícitas transmitidas culturalmente durante su proceso de formación.

Una aproximación al estudio de las teorías implícitas requiere considerar los aportes de diversos autores que han consolidado el ámbito de la comprensión textual (Duro, 1991; Goodman, 1994; Gough, 1994; Graesser, Singer \& Trabasso, 1994; Pearson \& Stephens, 1994; Rosenblatt, 1996), por cuanto esta investigación se plantea identificar las teorías que pudieran estar influyendo en las concepciones de los estudiantes respecto de este dominio del conocimiento. En consecuencia, las teorías desarrolladas en el ámbito de la comprensión nos permiten reconocer los enfoques y modelos que han tenido mayor impacto en definir y caracterizar los procesos de comprensión textual.

\section{CARACTERIZACION DE LAS TEORIAS IMPLICITAS SOBRE LA COMPRENSION TEXTUAL}

Las teorías implícitas sobre la comprensión de textos han sido sistematizadas de acuerdo a tres componentes centrales: noción de comprensión, de texto y de lector. A su vez, esta clasificación se ha realizado integrando información desde estudios historiográficos que sistematizan los diversos enfoques en el desarrollo de este concepto. Por otra parte, se integran en esta sistematización de teorías implícitas los datos obtenidos en diversos estudios cualitativos que permiten proponer en forma sintética los supuestos de cada teoría en torno a la noción de comprensión, de lector y de texto (ver tabla 1 en página siguiente).

Las teorías antes descritas representan una síntesis de los conocimientos acerca de la comprensión textual que forman parte de los enfoques sobre este ámbito de estudio.

\section{DISEÑO, METODOLOGIA Y PROCEDIMIENTOS DE INVESTIGACION}

Considerando que este estudio aborda las teorías implícitas sobre la comprensión textual de estudiantes universitarios (Makuc, 2004, 2008, 2009) se ha diseñado un cuestionario atribucional a partir de enunciados representativos de las teorías definidas en estudios historiográficos, focus group y entrevistas. Este proceso tiene la finalidad de extraer proposiciones y enunciados representativos de cada teoría sometidos a análisis de contenido (van Dijk \& Kintsch, 1983) y a la posterior determinación de tipicidad de los enunciados y verificación de la validez conceptual y lingüística de los ítems. 


\section{Tabla 1}

Síntesis de las teorías implícitas según la noción de comprensión lectora y texto (Makuc, 2004-208)

\begin{tabular}{|c|c|c|c|c|}
\hline & $\begin{array}{l}\text { Teoría } \\
\text { Lineal }\end{array}$ & $\begin{array}{c}\text { Teoría } \\
\text { Interactiva }\end{array}$ & $\begin{array}{c}\text { Teoría } \\
\text { Transaccional }\end{array}$ & $\begin{array}{c}\text { Teoría } \\
\text { Literaria }\end{array}$ \\
\hline $\begin{array}{c}\text { Noción } \\
\text { de } \\
\text { comprensión }\end{array}$ & $\begin{array}{l}\text { Comprender } \\
\text { es reproducir } \\
\text { el significado } \\
\text { del texto con la } \\
\text { mayor fidelidad. }\end{array}$ & $\begin{array}{l}\text { Comprender } \\
\text { es interactuar } \\
\text { con el texto, } \\
\text { construir el } \\
\text { significado } \\
\text { del texto a } \\
\text { partir de los } \\
\text { conocimientos } \\
\text { previos y las } \\
\text { experiencias. }\end{array}$ & $\begin{array}{l}\text { Comprender es } \\
\text { ser capaz de } \\
\text { compartir el } \\
\text { significado del } \\
\text { texto a través de } \\
\text { la comunicación } \\
\text { con otros. }\end{array}$ & $\begin{array}{l}\text { Comprender } \\
\text { es imaginar, } \\
\text { disfrutar, valorar } \\
\text { estéticamente } \\
\text { un texto. En } \\
\text { esta teoría, } \\
\text { comprender } \\
\text { implica } \\
\text { comparar } \\
\text { lecturas, } \\
\text { personajes, } \\
\text { identificar } \\
\text { características } \\
\text { asociadas a esos } \\
\text { personajes. }\end{array}$ \\
\hline $\begin{array}{c}\text { Noción } \\
\text { de } \\
\text { lector }\end{array}$ & $\begin{array}{l}\text { El lector debe } \\
\text { extraer el } \\
\text { significado del } \\
\text { texto. }\end{array}$ & $\begin{array}{l}\text { El lector es } \\
\text { activo, a través } \\
\text { de la lectura } \\
\text { integra los } \\
\text { significados del } \\
\text { texto con su } \\
\text { experiencia y } \\
\text { conocimientos. }\end{array}$ & $\begin{array}{l}\text { El lector en } \\
\text { la interacción } \\
\text { con los otros } \\
\text { construye el } \\
\text { significado del } \\
\text { texto. }\end{array}$ & $\begin{array}{l}\text { El lector disfruta } \\
\text { con la lectura, } \\
\text { se compromete, } \\
\text { es un lector } \\
\text { empático. }\end{array}$ \\
\hline $\begin{array}{l}\text { Noción } \\
\text { de } \\
\text { texto }\end{array}$ & $\begin{array}{l}\text { El texto } \\
\text { entrega toda } \\
\text { la información } \\
\text { necesaria para } \\
\text { la comprensión. } \\
\text { El vocabulario } \\
\text { del texto es } \\
\text { fundamental } \\
\text { para su } \\
\text { comprensión. }\end{array}$ & $\begin{array}{l}\text { El texto es } \\
\text { una parte } \\
\text { importante, pero } \\
\text { su significado } \\
\text { se completa } \\
\text { con el que el } \\
\text { lector es capaz } \\
\text { de asignarle. El } \\
\text { vocabulario no } \\
\text { es tan relevante. } \\
\text { Aspectos como } \\
\text { la coherencia } \\
\text { y la cohesión } \\
\text { facilitan la } \\
\text { comprensión. }\end{array}$ & $\begin{array}{l}\text { El texto debe ser } \\
\text { reestructurado, } \\
\text { transformado } \\
\text { y comunicado } \\
\text { en otras formas } \\
\text { orales o escritas. }\end{array}$ & $\begin{array}{l}\text { El texto debe } \\
\text { ser capaz de } \\
\text { emocionar, } \\
\text { debe tratar } \\
\text { temas de interés } \\
\text { del lector, } \\
\text { debe motivar } \\
\text { y provocar } \\
\text { cambios en el } \\
\text { lector, debe } \\
\text { provocar placer } \\
\text { y entusiasmo } \\
\text { en el lector, } \\
\text { debe invitar a la } \\
\text { lectura. }\end{array}$ \\
\hline
\end{tabular}


- Población: el universo de estudiantes universitarios de primer año de la Universidad de Magallanes asciende a 510 alumnos. Estos estudiantes corresponden a alumnos egresados de colegios municipalizados (261); colegios particulares subvencionados (200) y colegios privados (49).

\section{- Muestra}

En la presente investigación se identificaron las teorías implícitas predominantes y los niveles de competencias lectoras de estudiantes del Departamento de Educación. Los sujetos de la investigación fueron seleccionados a través de un tipo de mиеstreo no probabilístico. Específicamente se diseñó una muestra intencional de 90 alumnos de $1^{\text {er }}$ año, correspondiente del año 2009.

En cuanto a los aspectos metodológicos desarrollados con la finalidad de elicitar las teorías implícitas de los sujetos lectores existen diversas alternativas, sin embargo, en gran parte de estos estudios se consideran las siguientes etapas:

- Definición de teorías a partir de estudios historiográficos (Rodrigo, 2001).

- Una etapa exploratoria cualitativa, en la cual opcionalmente se realizan entrevistas (Castro, 2006).

- Sesiones de brainstorming grupales (Rodrigo, 2001), focus group (Makuc, 2004, 2008), verbalización de percepciones (Estrada, 2004).

Cada una de estas etapas tiene la finalidad de extraer proposiciones (aseveraciones sobre un determinado aspecto del fenómeno en estudio) que sean representativas de cada teoría que se somete a los respectivos análisis de contenido y a la posterior determinación de tipicidad de los enunciados y verificación de la validez conceptual y lingüística de los ítems.

Posteriormente, en una etapa cuantitativa se diseñan, validan y aplican instrumentos de medición de diverso tipo: cuestionarios atribucionales (Marrero, 1988; Rodrigo, 1993; 1988; Jiménez, 1999); cuestionarios que describen atributos (Hanges, 2004; Castro, 2006); cuestionarios que describen percepciones (Estrada, Yzerbit \& Serón, 2004; Makuc, 2009). En este contexto, cabe señalar que la realización de este proceso de redescripción se enmarca en el supuesto de que la explicitación es un proceso gradual. Y, por otra parte, se sostiene que en todo conocimiento hay componentes implícitos y explícitos, por ello, tanto la verbalización como el reconocimiento nos permiten acceder a las teorías que los sujetos sostienen sobre un determinado dominio (Karmiloff Smith, 1994; Pozo, 2001). La figura 1, en la página siguiente, explicita esta propuesta: 
Figura 1

Esquema de la redescripción representacional (Basado en Karmiloff Smith, 1994)

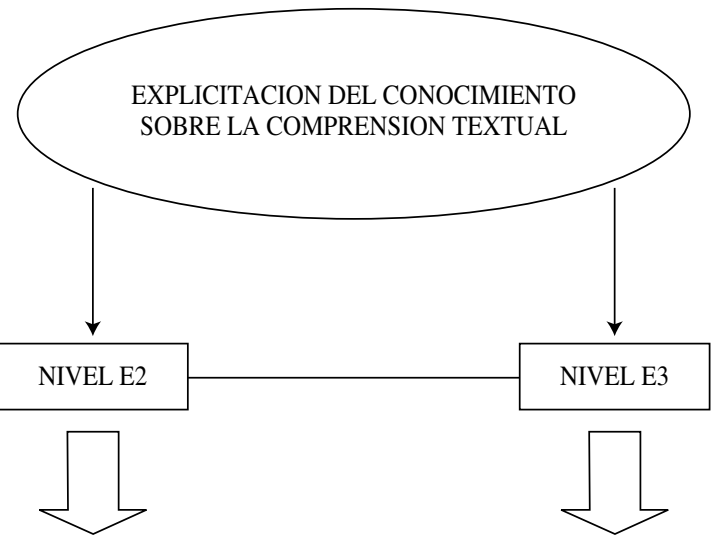

Nivel de Reconocimiento

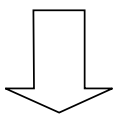

Cuestionario de Teorías Implícitas
Nivel de Verbalización

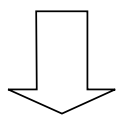

Focus Group-Entrevistas

\section{- $\quad$ Instrumentos de recolección de datos}

Como herramientas de recolección de datos se utilizaron dos instrumentos: a) Batería de pruebas de comprensión de textos (Parodi, 2005) y b) Cuestionario de Teorías Implícitas sobre la Comprensión de Textos (Makuc, 2009). Cada uno de estos instrumentos se describe a continuación.

A. Batería de pruebas de comprensión de textos (Parodi, 2005b).

Esta batería de pruebas ha sido elaborada y aplicada con la finalidad de medir las habilidades lectocomprensivas en el marco del proyecto Fondecyt (1020786). Cada una de las pruebas consta de dos partes: un texto escrito y un conjunto de 13 preguntas abiertas de tipo inferencial; este tipo de preguntas exige superar el nivel de reconocimiento y memorización de la información textual. Los niveles de comprensión textual que organizan las pruebas son: Nivel microestructural: preguntas de inferencia léxica y causal; Nivel macroestructural: preguntas de inferencia temática y causal transpárrafo y Nivel superestructural: preguntas de inferencia superestructural de acuerdo al tipo de texto. La importancia de aplicar esta batería de pruebas se relaciona con el hecho de que los textos están seleccionados para estudiantes de enseñanza secundaria del área humanística, científica y técnica, y los estudiantes universitarios de primer año provienen de diversas áreas de formación, lo que disminuye la posibilidad de que algunos estudiantes obtengan menores resultados debido a su especialidad. Este instrumento de 
evaluación consta de preguntas con puntajes diferenciados según el grado de dificultad de acuerdo al nivel o al tipo de procesamiento. En la tabla siguiente se presentan los tipos, la cantidad y los porcentajes asociados a cada grupo de preguntas.

Tabla 2

Tipo de preguntas y puntajes asignados

\begin{tabular}{|c|c|c|c|c|}
\hline Tipo de pregunta & Subtipo & $\mathrm{N}^{\circ}$ preguntas & Puntaje máximo & Porcentajes \\
\hline Literal-Local & & 4 & 12 & 8,7 \\
\hline Inferencia local & $\begin{array}{l}\text { Léxica } \\
\text { Correferencial } \\
\text { Causa-efecto }\end{array}$ & $\begin{array}{l}2 \\
2 \\
2\end{array}$ & 36 & 26,1 \\
\hline Inferencia global & $\begin{array}{l}\text { Idea Global } \\
\text { Causa-efecto } \\
\text { Título } \\
\text { Resumen }\end{array}$ & $\begin{array}{l}1 \\
1 \\
1 \\
1\end{array}$ & 70 & 50,7 \\
\hline Aplicación & & 1 & 20 & 14,5 \\
\hline Totales & & 15 & 138 & 100 \\
\hline
\end{tabular}

B. Cuestionario de Teorías Implícitas sobre la Comprensión de Textos (Makuc, 2009)

Este cuestionario plantea cuatro teorías implícitas sobre la comprensión de textos: teoría lineal, interactiva, transaccional y literaria. Estas teorías sostienen concepciones diversas acerca de tres temas centrales en la comprensión textual: noción de texto, de lector y de comprensión. El cuestionario consta de seis enunciados prototípicos de cada teoría, la validez y consistencia interna de los constructos se ha realizado entre estudiantes universitarios y expertos en el área de lenguaje en el marco del Proyecto de Investigación del año 2008 de la Dirección de Investigación (UMAG) (No PYF4-01EH-08-09). La estructura de este instrumento se presenta en la tabla 3.

\section{Tabla 3}

Distribución de preguntas de acuerdo a cada teoría implícita

\begin{tabular}{|l|l|c|}
\hline Tipo de pregunta & Preguntas & Puntaje máximo \\
\hline Teoría Lineal & $1-5-10-14-19-20$ & 24 \\
\hline Teoría Interactiva & $2-8-13-17-18-21$ & 24 \\
\hline Teoría Literaria & $3-7-11-15-16-22$ & 24 \\
\hline Teoría Transaccional & $4-6-9-12-23-24$ & 24 \\
\hline Totales & 24 & 96 \\
\hline
\end{tabular}




\section{PRESENTACION Y DISCUSION DE RESULTADOS}

Los resultados que se presentan a continuación corresponden a los obtenidos en la aplicación de dos instrumentos: Cuestionario de Teorías Implícitas y Batería de pruebas de comprensión de textos.

\subsection{RESULTADOS DEL CUESTIONARIO DE TEORIAS IMPLICITAS}

Los resultados obtenidos para cada una de las Teorías Implícitas de la Comprensión de textos se pueden ver en la tabla 4 de porcentajes.

\section{Tabla 4}

Clasificación de los estudiantes de acuerdo a la teoría predominante

\begin{tabular}{|l|c|c|}
\hline Teoría Implícita & Porcentaje de estudiantes & Número de estudiantes \\
\hline Lineal & $22,6 \%$ & 17 \\
\hline Interactiva & $33,3 \%$ & 25 \\
\hline Transaccional & $14,6 \%$ & 11 \\
\hline Literaria & $29,3 \%$ & 22 \\
\hline Total & $99,8 \%$ & 75 \\
\hline
\end{tabular}

En esta tabla se puede observar que la mayoría de los estudiantes (75) se distribuyen en alguna de las cuatro teorías consultadas: Lineal, Interactiva, Transaccional y Literaria (75). Sin embargo, entre ellos la teoría implícita predominante es la teoría interactiva $(33,3 \%)$ seguida por la teoría literaria $(29,3 \%)$, observándose, al mismo tiempo que la teoría con menor adhesión es la teoría transaccional $(14,6 \%)$. El porcentaje restante de estudiantes $(17,6 \%)$ adhiere a lo que hemos definido como teorías híbridas, lo que se expresa en que obtienen el mismo puntaje en dos teorías: lineal/literaria; lineal/transaccional; Literaria/Interactiva; Literaria/Transaccional.

\subsection{RESULTADOS DE LA APLICACION DE PRUEBAS DE COMPRENSION TEXTUAL}

La obtención de estos resultados se logró a partir de la aplicación de tres pruebas de comprensión (Parodi, 2005b). Estos resultados fueron analizados en dos niveles: resultados generales obtenidos por los sujetos en la prueba de comprensión y resultados específicos por grupos y por tipos de pregunta. Considerando que para evaluar el rendimiento se ha fijado en $60 \%$ el nivel de logro aceptable, los resultados obtenidos por los 75 estudiantes que adhieren a las teorías implícitas en estudio se organizan en la tabla 5.

En la tabla 5 se puede observar que sólo el $56 \%$ de los jóvenes obtiene logros satisfactorios, mientras que el $44 \%$ no alcanza los niveles mínimos de rendimiento. Estos deficientes resultados del grupo de estudio serán analizados en función de los niveles de comprensión medidos por las pruebas. 
Tabla 5

Resultados obtenidos en la aplicación de pruebas de comprensión textual

\begin{tabular}{|l|c|c|}
\hline Rango/Puntaje & $\mathrm{N}^{\circ}$ de estudiantes (75) & Porcentaje de estudiantes \\
\hline Sobre 60\% de logro & 42 & $56 \%$ \\
\hline Bajo 60\% de logro & 33 & $44 \%$ \\
\hline Total & 75 & $100 \%$ \\
\hline
\end{tabular}

Tabla 6

Resultados específicos por tipo de pregunta

\begin{tabular}{|l|l|c|c|}
\hline Tipo de pregunta & \multicolumn{1}{|c|}{ Subtipo } & Sobre 60\% de logro & Bajo 60\% de logro \\
\hline Literal-local & & $56,82 \%$ & $43,18 \%$ \\
\hline Inferencia local & $\begin{array}{l}\text { Léxica } \\
\text { Correferencial } \\
\text { Causa-efecto }\end{array}$ & $18,94 \%$ & $81,06 \%$ \\
\hline Inferencia global & $\begin{array}{l}\text { Idea Global } \\
\text { Causa-efecto } \\
\text { Título } \\
\text { Resumen }\end{array}$ & $35,23 \%$ & $64,77 \%$ \\
\hline Aplicación & & $18,18 \%$ & $81,82 \%$ \\
\hline
\end{tabular}

Respecto de los resultados específicos por tipos de pregunta observamos los siguientes datos (ver tabla 6).

En la tabla anterior es posible comprobar que los resultados por tipo de pregunta están estrechamente relacionados con el grado de exigencia que enfrenta el lector. En el nivel local, los estudiantes obtienen los mejores resultados, nivel que corresponde a una primera dimensión literal, es decir, al nivel superficial del texto clásicamente caracterizado como de menor exigencia en que el sujeto debe reconocer y reproducir información, generalmente explícita. Este rendimiento disminuye drásticamente, cuando se supera el nivel literal-explícito y se proponen preguntas en que el sujeto debe inferir información implícita: de este modo en el nivel inferencial (léxica, correferencial y causal) apenas un $18 \%$ de sujetos alcanza un nivel de logro satisfactorio, resultado muy similar al obtenido en la pregunta de aplicación. En relación a la inferencia global se observa un mejor resultado, pues el 35\% de los estudiantes resuelve exitosamente este tipo de preguntas. La última pregunta, denominada de Aplicación, los estudiantes presentan los resultados más bajos $(18,18 \%)$, esta pregunta exigía generar una inferencia proyectiva o elaborativa y requiere que el lector utilice activamente conocimientos adquiridos en el contexto de la comprensión del texto, lo que explicaría la dificultad enfrentada por los estudiantes. 


\section{Tabla 7}

Porcentaje de logro obtenido por los estudiantes que adhieren a las teorías implícitas del estudio

\begin{tabular}{|l|c|c|c|c|}
\hline Porcentaje de Logro & Teoría Lineal & Teoría Interactiva & Teoría Transaccional & Teoría Literaria \\
\hline Sobre 60\% de logro & $64,7 \%$ & $76 \%$ & $54,5 \%$ & $54,5 \%$ \\
\hline Bajo 60\% de logro & $35,2 \%$ & $24 \%$ & $45,4 \%$ & $45,4 \%$ \\
\hline Total & $99,9 \%$ & $100 \%$ & $100 \%$ & $100 \%$ \\
\hline
\end{tabular}

\subsection{RELACION ENTRE LOS RESULTADOS DEL CUESTIONARIO DE TEORIAS IMPLICITAS Y RESULTADOS OBTENIDOS EN NIVELES DE COMPETENCIA LECTORA}

Como una forma de integrar los datos provenientes de la aplicación de los dos instrumentos principales de este estudio, a continuación se presenta la información que permite sostener una relación entre ambas dimensiones del estudio de la comprensión de textos en estudiantes universitarios: a) Dimensión de las teorías implícitas b) Dimensión de competencia lectora.

Los datos obtenidos sobre la relación entre ambas dimensiones del estudio son los siguientes (ver tabla 4).

En esta tabla podemos observar los resultados obtenidos en la prueba de comprensión de los estudiantes distribuidos en las teorías implícitas del estudio. Con respecto a los resultados de los alumnos que sostienen una teoría lineal, el 64,7\% de ellos obtiene un buen rendimiento, sin embargo, un $35,2 \%$ de los estudiantes que adhieren a esta teoría obtiene resultados insuficientes. Por su parte, los estudiantes que adhieren a una teoría literaria y transaccional presentan un $54,5 \%$, con un nivel de logro suficiente, y un $45,4 \%$ de ellos no alcanza niveles mínimos de comprensión. Se observa una diferencia con el grupo de estudiantes que sostienen una teoría interactiva, pues en este grupo el $76 \%$ obtiene logros satisfactorios y el $24 \%$ presenta logros insatisfactorios.

En términos generales, es posible observar dos teorías implícitas predominantes: Teoría Implícita Literaria y Teoría Implícita Interactiva (Tabla 3); a su vez, los estudiantes que adhieren a estas teorías presentan diferencias respecto de los grados de competencia lectora obtenidos en las pruebas de comprensión: el 54,5\% de aquellos que adhieren a la Teoría Literaria obtiene logro satisfactorios; mientras que aquellos estudiantes que adhieren a la Teoría Implícita Interactiva el 76\% de ellos logra resultados sobre un $60 \%$ de logro. Lo anterior nos muestra un logro superior de rendimiento en el grupo que sostiene la Teoría Interactiva. A su vez, podemos observar que el nivel inferior de rendimiento es obtenido por los estudiantes que adhieren a la Teoría Literaria y Transaccional.

\section{CONCLUSIONES}

En estos resultados se ha logrado identificar dos aspectos de la comprensión lectora de estudiantes que ingresan a la educación superior; por una parte, se observa que un 
número importante de estudiantes no obtiene resultados satisfactorios, particularmente en el nivel de inferencia local y de aplicación, evidenciando al igual que otros estudios en estudiantes universitarios (Ibáñez, 2008) que éstos no logran generar una base textual del texto. Específicamente, en el caso de la aplicación, el lector debe utilizar activamente conocimientos adquiridos en el contexto de la comprensión del texto; este nivel plantea ir más allá de la información textual, generando un modelo de situación por el lector. En otras palabras, el lector debe relacionar, aplicar y proyectar lo aprendido a partir del texto a una situación novedosa, dentro del marco de la lectura del texto.

Por su parte, en la indagación acerca de las teorías implícitas que predominan en lectores con distinto nivel de experticia, hemos obtenido como información que las teorías que mayor adhesión concitan entre los estudiantes con mayor experticia corresponden a la teoría implícita interactiva y lineal, mientras que los estudiantes con más bajos niveles de experticia adhieren a la teoría literaria y transaccional.

Dado que este estudio plantea que las teorías implícitas estarían incidiendo en la experticia, en términos generales se observa que los estudiantes que adhieren a la teoría implícita interactiva obtienen mayoritariamente mejores resultados que los sujetos que adhieren a las restantes teorías implícitas. Al mismo tiempo y considerando las teorías que concitan mayor adhesión (Teoría implícita Interactiva y Literaria) la teoría interactiva obtiene mejores resultados, a diferencia de los estudiantes que adhieren a la teoría implícita literaria en la cual un porcentaje mayor de sujetos $(45,4 \%)$ no supera los requerimientos mínimos de la evaluación aplicada.

Es importante destacar que entre los supuestos de la teoría interactiva (Tabla 1) los lectores se caracterizan por ser activos, completan la información del texto realizando inferencias en los distintos niveles; este tipo de lector no se orienta a reproducir información literal, sino que confía en sus capacidades para identificar la coherencia del texto. Las características de los lectores que adhieren a este tipo de planteamientos fueron recogidas en los enunciados del cuestionario y refieren a la teoría implícita interactiva. Los estudiantes que adhieren a este tipo de planteamientos pueden caracterizarse como lectores interactivos y obtienen mayoritariamente resultados favorables.

Por su parte, los estudiantes que sostienen una teoría literaria adhieren a planteamientos que se caracterizan por concebir la comprensión desde una perspectiva más bien estética y de búsqueda de placer, en donde comprender se asocia con imaginar, disfrutar, valorar estéticamente un texto. Entre este tipo de lectores al ser evaluados en su experticia lectora encontramos que la mayor cantidad de estudiantes se encuentra en el rango de resultados deficitarios $(54,5 \%)$ y un menor número de ellos alcanza resultados satisfactorios $(45,5 \%)$.

Estos resultados permiten destacar la importancia de realizar una caracterización de lector (tipología del lector) no sólo desde la perspectiva de la competencia lectora (lector competente, experto/vs inexperto), sino, además, desde la perspectiva de las teorías implícitas, esto es, reconociendo que todo lector adhiere a ciertos planteamientos, concibe la lectura de un cierto modo, se ubica desde un punto de vista determinado frente a la lectura, al texto, al rol del lector, es decir, tiene una representación de la comprensión que moviliza sus acciones, de manera implícita, orientándolo respecto de cómo dirigir su atención y canalizar sus recursos cognitivos (conocimientos declarativos y procedimentales) hacia determinadas metas que desde su concepción resultan adecuadas y pertinentes. 
De este modo, abordar este nivel del conocimiento implícito del lector, adquirido mediante la experiencia y formación, permite caracterizar las formas de aproximación del lector hacia la comprensión textual, explicitar sus fundamentos, identificar sus componentes, en otras palabras, mostrar al propio lector sobre qué supuestos aborda la comprensión textual y cuáles de estos enfoques resultan más adecuados cuando la tarea es enfrentar el texto con fines académicos que exigen competencia lectora.

En este sentido, las teorías implícitas sobre la lectura pueden entregarnos información útil, no sólo para que el lector reconozca su perfil lector, sino que en procesos de intervención cabe explicitar, analizar y profundizar en las teorías implícitas que orientan las decisiones del lector como parte integral de las estrategias dirigidas a desarrollar la experticia lectora en los estudiantes. Finalmente, es preciso destacar que estos resultados deben considerarse preliminares, en tanto constituyen la posibilidad de avanzar y profundizar en las características de los estudiantes que sostienen diversas teorías, explicitando, por ejemplo, qué teoría se activa en los diversos niveles de complejidad de los textos o frente a determinadas inferencias en qué variable se concentra una determinada teoría implícita: en la noción de comprensión, de texto, de lector. En síntesis, la noción de teoría implícita nos introduce en un ámbito que al profundizarse orienta futuras estrategias de intervención (González, 2008) tendientes a enfrentar no sólo las deficiencias en el ámbito de la comprensión textual, sino además reconocer las características del lector experto, desde diversas miradas, que nos indican que la competencia lectora es un complejo de múltiples procesos, en donde la experiencia y los modelos culturales cumplen un importante rol.

\section{REFERENCIAS BIBLIOGRAFICAS}

Alonso, J. (2005). Claves para la enseñanza de la comprensión lectora. Revista de Educación ( $\mathrm{N}^{\mathrm{o}}$ extraordinario): 63-93.

Hernández, G. (2008) Teorías implícitas de lectura y conocimiento metatextual En: Revista Mejicana de Investigación Educativa, 13,38, 737-771.

Bravo, D. \& Contreras, D. (2001). Competencias básicas de la población adulta, Chile: Universidad de Chile, Departamento de Economía.

Brunner, J.J. \& Elacqua, B. (2003). Informe de Capital Humano en Chile, Santiago: Universidad Adolfo Ibáñez.

Carey. S. \& Spelke, E. (1993). Domain specific knowledge and conceptual change. En Goldman, S. y Hirschfeld, L. (eds.), Cultural knowledge and domain specificity. New York: Cambridge University Press.

Castro, A. (2006). Teorías implícitas del liderazgo, contexto y capacidad de conducción. Anales de Psicología, vol. 22, № 1, 89-97.

Colomer, T. \& A. Camps (1996). Enseñar a leer, enseñar a comprender. Madrid: Celeste Ediciones.

Dann, H. (1990). Subjective Theories: A New Approach to Psychological Research and Educational Practice. En Semin, G. \& Gergen, K. (eds.), Everyday Understanding (pp. 227-243). London: Sage Publications.

De Vega, M. (2002). Del significado simbólico al significado corpóreo. Estudios de Psicología, 23, 153-174.

Dienes, Z. \& Perner, J. (1999). A theory of implicit and explicit knowledge. Behavioral and Brain Sciences. 22: 735-808.

Duro, A. (1991). La coherencia textual en los modelos para la comprensión de textos: Kintsch et al. Vs. Sanford y Garrod. Cognitiva. 4, 2: 226-242. 
Estrada, C., Yzerbit, V. \& Serón, E. (2004). El efecto del esencialismo psicológico sobre las teorías ingenuas de las diferencias grupales. Psicothema, 16 (2), 181-186.

Eyzaguirre, B. \& Fontaine, L. (2008). Las Escuelas que Tenemos, Santiago, Centro de Estudios Públicos.

Furnham, A. (1988). Lay Theories. Everyday understanding of problems in social sciences. New York: Pergamon Press.

Furnham, A. (1990). Commonsense Theories of Personality. En Semin, G. \& Gergen, K. (eds.), Every Day Understanding. Social and Scientific Implications (pp. 176-203). London: Sage.

González, K. (2008). "Propuesta de un programa para mejorar la comprensión de textos en estudiantes universitarios", en Revista Electrónica, Instituto de Investigación en Educación, Universidad de Costa Rica, 8 (2) pp. 1-31. http://.revista.inie.ucr.ac.cr

Goodman, K. (1994). Reading, writing, and written texts: A Transactional Sociopsycholinguistic View. En Theoretical models and processes of reading (pp. 1093-1130). Newark, Delaware: IRA \& Erlbaum.

Gough, P. (1994). One second of reading, en Singer, H. y Rudell, B. (eds.), Theoretical models and processes of reading (pp. 661-686). Newark, Delaware: IRA \& Erlbaum.

Graesser, A.C., Singer, M. \& Trabasso, T. (1994). Constructing inferences during narrative text comprehension. Psychological Review 101: 371-395.

Groeben, N. (1990). Subjective Theories and the Explanation of Human Action. En Semin, G.R. \& Gergen, K. (eds.), Every Day Understanding. Social and Scientific Implications (pp. 1944). London: Sage.

Hanges, P. (2004). Research Methodology. En House, R.J., Hanges, P.J., Javidan, M., Dorfman, P. \& Gupta, V. (eds.) Leadership, culture and organization: The Globe study of 62 societies (pp. 91-101). London: Sage Publications.

Jamet, E. (2006). Lectura y éxito escolar. Buenos Aires: Fondo de Cultura Económica.

Ibáñez, R. (2008). Comprensión de textos académicos escritos en inglés: relación entre nivel de logro y variables involucradas. Revista Signos, 41 (67) 203-229.

Jiménez, A. (2002). Nivel educativo y teorías implícitas sobre la enseñanza: estructura de creencias en los docentes de educación infantil y primaria, secundaria y superior. Tesis Doctoral. Pontificia Universidad Católica de Santiago.

Karmiloff-Smith, A. (1994). Más allá de la modularidad. Madrid: Alianza.

Karmiloff-Smith, A. (1997). Autoorganización y cambio cognitivo. Substratum, 1 (1), 19-43.

Kihlstrom, J. (1999). Conscious Versus Unconscious Cognition. En Sternberg, R. (ed.), The nature of Cognition (pp. 173-241). Cambridge: MIT.

Kintsch, W. (1994). The role of Knowledge in Discourse Comprehension: A Construction-Integration Model. En Singer, H. \& Rudell, B. (eds.). Theoretical models and processes of reading (951995). Newark, Delaware: IRA \& Erlbaum.

Kintsch, W. \& van Dijk, T. (1978). "Toward a model of text comprehension and production”, en Psychological Review 85 (5), 363-394.

Kintsch, W. (2002). On the notions of theme and topic in psychological process models of text comprehension. En Louwerse, M. \& van Peer, W. (eds.), Thematics: Interdisciplinary studies (pp. 151-170). Amsterdam: Benjamins.

Laberge, D. \& Samuels, S. (1994). Toward a theory of automatic information processing of reading. En Singer, H. y Rudell, B. (eds.). Theoretical models and processes of reading (pp. 689-718). Newark, Delaware: IRA y Erlbaum.

Long, D. \& Chong, J. (2001). Comprehension Skill and Global Coherence: A Paradoxical Picture of Poor Comprehenders' Abilities". Journal of Experimental Psychology: Learning, Memory and Cognition. $\mathrm{N}^{\circ}$ 6: 1424-1429.

Makuc, M. (2004). Teorías implícitas de los profesores acerca de la comprensión de textos. Tesis para optar al grado de Doctor en Lingüística por la Pontificia Universidad Católica de Valparaíso. 
Makuc, M. (2008). Teorías implícitas de los profesores acerca de la comprensión de textos. Revista Signos, 41, 68, 403-422.

Makuc, M. (2009). Estudio sobre la comprensión de textos en estudiantes de primer año de la Universidad de Magallanes. Revista Ciencia en el Austro. Estudios e Investigaciones desarrollados por la Universidad de Magallanes, 4, 109-118.

Makuc, M., Judikis, J., Estrada, C. y Molina, W. (2009). Teorías implícitas sobre el aprendizaje y su relación con las prácticas pedagógicas: un estudio de caso. Selección de Investigaciones Primer Concurso FONIDE: Evidencias para políticas públicas en Educación. Fondo de Investigación y Desarrollo en Educación. Santiago: MINEDUC.

Marrero, J. (1988). Las teorías implícitas y la planificación del profesor. Tesis Doctoral no publicada. Universidad de la Laguna. España.

Mateos, M. (2001). Metacognición y Educación. Buenos Aires: Aique.

Maturano, C., Mazzitelli, C. y Macias, A. (2006). ¿Cómo los estudiantes regulan la comprensión cuando leen un texto instructivo con dificultades? Revista Electrónica de Enseñanza de las Ciencias. Vol. 2, No 1, 235-246.

McKoon, G. \& Ratcliff, R. (1998). Memory-based Language Processing: Psycholinguistic Research in the 1990s. Annual Review of Psychology, 49: 25-42.

Mineduc (2003a). Desempeño de los estudiantes chilenos: Resultado de PISA. Departamento de Estudios y Estadísticas de la División de Planificación y Presupuesto del Ministerio de Educación.

Mitchell, J. (1995). Teacher's Implicit Theories Concerning Questioning. British Educational Research Journal. 1: 69-84.

Murphy, G. \& Medin, D. (1985). "The role of theories in conceptual coherence". Psychological Review. 3: 289-316.

OECD (2010). Estudio económico de Chile. París: Organization for Economic Cooperation and Development.

OECD (2000). Literacy in the Information Age. Paris: Organization for Economic Cooperation and Development. Statistics Canadá.

OECD (2003). Proyecto PISA Competencias en lectura. [En línea]. Disponible en http://www.pisa. oecd.org.

OECD (2006). Evaluación Educativa, resumen ejecutivo. [en línea]. Disponible en http://www.oei.es

Paris, S., Lipson M. \& Wixson K. (1994). Becoming a Strategic Reader. En Singer, H. \& Rudell, B. (eds.). Theoretical Models and processes of reading (pp. 778-862). Newark, Delaware: IRA.

Parodi, G. (2002). Comprensión lingüística: ¿Hacia dónde vamos desde donde estamos? En Parodi, G. (ed.), Lingüística e interdisciplinariedad: Desafíos del nuevo milenio. Ensayos en honor a Marianne Peronard (pp. 67-93), Valparaíso: Ediciones Universitarias de Valparaíso.

Parodi, G. (2003). Relaciones entre lectura y escritura: una perspectiva cognitiva discursiva. Bases teóricas y antecedentes empíricos, Valparaíso: Ediciones Universitarias de Valparaíso.

Parodi, G. (2005a). Comprensión de textos escritos, Buenos Aires: Eudeba.

Parodi, G. (2005b). La comprensión del discurso especializado escrito en ámbitos técnico-profesionales: ¿aprendiendo a partir del texto? Signos, 38, (58) 221-267.

Parodi, G. (2007). Comprensión y aprendizaje a partir del discurso especializado escrito: Teoría y empiria. En Parodi, G. (ed.), Lingüística de corpus y discursos especializados: Puntos de mira (pp. 223-258). Valparaíso: Ediciones Universitarias de Valparaíso.

Pearson, D. \& Stephens D. (1994). Learning about Literacy: A 30-Year Journey. En Singer, H. \& Rudell, B. (eds.), Theoretical Models and processes of reading (pp. 22-103). Newark, Delaware: IRA \& Erlbaum.

Peronard, M., Crespo, N. \& Velázquez, M. (2000). La evaluación del conocimiento metacomprensivo en alumnos de educación básica. Signos, XXXIII, 47: 167-180.

Peronard, M., Velázquez, M., Crespo, N. \& Viramonte, M. (2002). Conocimiento Metacognitivo del Lenguaje Escrito: Instrumento de Medida y Fundamentación Teórica. Infancia y Aprendizaje, 25 (2): 131-145. 
Peronard, M. (2005). La metacognición como herramienta didáctica. Revista Signos, 38 (57) $61-74$.

Peronard, M. (2007). Lectura en papel y en pantalla de computador. Revista Signos, 40 (63) 179-195.

Pozo, J. (2001). Humana mente. El mundo, la conciencia y la carne. Madrid: Morata.

Reber, A., Allen, R. \& Reber, P. (1999). Implicit versus Explicit Learning. En Sternberg, R. (ed.), The Nature of Cognition (pp. 475-505). Cambridge: MIT.

Regueiro, M. (2005). "Técnicas de comunicación en el aula". En Chamorro, M. \& Sánchez, P. (Coord.). Iniciación a la docencia universitaria. Manual de ayuda (pp. 179-207), Madrid: ICE de la Universidad Complutense.

Rinck, M., Gómez, E., Díaz, J. \& de Vega, M. (2003). Processing of Temporal Information: Evidence from Eye Movements. Memory and Cognition, 31, 77-86.

Riquelme, G., Cerda, G. \& Contenla, J. (2007). Test de habilidades lectocomprensivas básicas "THLB versión II".

Rivera, M. (2000). Estrategias de lectura para la comprensión de textos escritos: el pensamiento reflexivo y no lineal en alumnos de educación superior. Revista Digital Umbral, 12, 1-6.

Rodrigo, M.J. (1985). Las teorías implícitas en el conocimiento social. Revista Infancia y Aprendizaje, 31-32: $145-156$.

Rodrigo, M.J. (1993). Representaciones y procesos en las teorías implícitas. En Rodrigo, M.J., Rodríguez, A. y Marrero, J. (eds.). Las teorías implícitas. Una aproximación al conocimiento cotidiano (pp. 95-117) Madrid: Visor.

Rodrigo, M.J. (1994b). Etapas, contextos, dominios y teorías implícitas en el conocimiento social. En Rodrigo, M.J. (ed.). Contexto y desarrollo social. Madrid: Síntesis.

Rodrigo, M.J. (1997). Del escenario sociocultural al constructivismo episódico: un viaje al conocimiento escolar de la mano de las teorías implícitas. En Rodrigo, M. y Arnay, J. (compiladores). La construcción del conocimiento escolar. Ediciones Paidós, Barcelona, 1997.

Rodrigo, M.J. \& Correa, N. (2001). Representación y procesos cognitivos: esquemas y modelos mentales. En Coll, C., Palacios, J. y Marchesi, A. (Comps.). Desarrollo psicológico y educación, 2. Psicología de la educación escolar (pp. 117-135). Madrid: Alianza.

Rodríguez, E. (2001). Teoría implícita y formación inicial del profesorado de Educación Media. Revista Enfoques Educacionales, 3, 2: 145-155.

Rosenblatt, L. (1996). La teoría transaccional de la lectura y la escritura. En Textos en contexto. Los procesos de lectura y escritura. Buenos Aires: Proyecto Editorial Lectura y Vida (pp. 13-70).

Rumelhart, D. (1997). Hacia una comprensión de la comprensión. En Rodríguez, E. y Laguer, E. (comp.), La lectura, Santiago de Cali: Editorial Universidad del Valle (pp. 25-51).

Schraw, G. \& Bruning, R. (1996). Readers' implicit models of reading. Reading Research Quarterly, 31, 3: 290-305.

Schraw, G. (2000). Reader beliefs and meaning construction in narrative text. Journal of Educational Psychology, 92, 1, pp. 96-106.

Silvestri, A. (2006). La formulación de preguntas para la comprensión de textos: estudio experimental. Revista Signos, 39, 62, 493-510.

SIMCE (2008) Evaluaciones internacionales [en línea]. Disponible en: http://www.simce.cl/paginas/ evaluaciones.htm

Smith, F. (1983). Comprensión de la lectura. Análisis psicolingüístico de la lectura y su aprendizaje, México: Trillas.

Van Dijk T. \& Kintsch, W. (1983). Strategies of discourse comprehension. New York: Academic Press.

Van Dijk, T. (1995). "On macrostructures, mental models and others inventions: a brief, personal history of the Kintsch-van Dijk Theory", en Weaver, C., Mannes, S. \& Fletcher, C. (eds.). Discourse comprehension: Essays in honor of Walter Kintsch (pp. 383-407). Mahwah, N.J., Erlbaum.

Van Dijk, T. (1999). Context models in discourse processing. En van Oostendorp, H., Goldman, S. (eds.), The construction of model representations during reading (123-146). Mahwah, N.J.: Erlbaum.

Van Dijk, T. (2001). Algunos principios de la teoría del contexto. Revista Latinoamericana de Estudios del Discurso, 1 (1), 69-82. 